\title{
Caracteres pre-faena de novillos tipos Braford y Brangus del nordeste argentino
}

\author{
Capellari, A.'; Rébak, G.I.'; Ynsaurralde, A.E. ${ }^{2}$; Yostar, E.J.'; Yostar, M.M. ${ }^{1}$
}

${ }^{1}$ Cátedra Producción Bovina, ${ }^{2}$ Cátedra Tecnología de los Alimentos, Facultad de Ciencias Veterinarias, UNNE, Sargento Cabral 2139, Corrientes (Argentina). E-mail: acapellari@vet.unne.edu.ar.

\begin{abstract}
Resumen
Capellari, A.; Rébak, G.I.; Ynsaurralde, A.E.; Yostar, E.J.; Yostar, M.M.: Caracteres pre-faena de novillos tipos Braford y Brangus del nordeste argentino. Rev. vet. 24: 1, 39-43, 2013. El objetivo del trabajo fue evaluar la influencia del tipo de alimentación (pastizal natural vs pastura cultivada) y de la edad aproximada (establecida por el número de dientes definitivos) en la producción de dos tipos de novillos (Braford y Brangus), a través de variables evaluadas en animales en pie previamente a la faena, en cuatro zonas del nordeste argentino. En base a las características del suelo, clima, vegetación y actividad productiva, los establecimientos seleccionados para la investigación correspondieron a varias "zonas agroeconómicas homogéneas" (ZAH). Se incluyeron establecimientos de la Provincia de Corrientes (departamentos de General Paz en la ZAH I y de Santo Tomé en la ZAH III), de Formosa (Departamento Patiño en la ZAH 8) y de Chaco (Departamento Independencia en la ZAH 2). Al finalizar el engorde se evaluaron los resultados obtenidos sobre 176 novillos de ciclo largo a través de parámetros como peso final individual medido por balanza, condición corporal (CC: escala 1-9), área de ojo de bife (AOB) y espesor de grasa dorsal (EGD) por la técnica de ultrasonido. El análisis de la varianza incluyó los efectos tipo, alimentación y "boqueo" (2, 4, 6 y 8 dientes definitivos), estableciéndose un riesgo alfa del $5 \%$. Las diferencias de los pesos de faena no fueron significativas entre los tipos y sistemas de alimentación. Los novillos del tipo Brangus finalizaron el engorde con una condición corporal más alta y los tipo Braford con un área de ojo de bife y un espesor de grasa dorsal superiores, en ambos casos estadísticamente significativas en los novillos de 2 a 4 años de edad criados sobre pastura cultivada.
\end{abstract}

Palabras clave: novillo, edad, alimentación, peso de faena, área de ojo de bife, grasa dorsal.

\begin{abstract}
Capellari, A.; Rébak, G.I.; Ynsaurralde, A.E.; Yostar, E.J.; Yostar, M.M.: Pre-slaughter characters of Braford and Brangus steer types from northeastern Argentina. Rev. vet. 24: 1, 39-43, 2013. The objectives of the assay were to evaluate the influence of the feeding system (natural grassland vs cultivated pasture) and the approximate age (estimated by number of permanent teeths) on the production of two types of steers (Braford and Brangus), through variables evaluated in vivo previous to the slaughter of animals in four areas of northeastern Argentina. Considering the characteristics of soil, climate, vegetation and productive activity, the farms selected for this trial corresponded to several "homogeneous agroeconomical areas" (HAA). Farms from the Province of Corrientes (General Paz in the HAA I and Santo Tome in the HAA III departments, respectively), Formosa (Patiño in the HAA 8 department) and Chaco (Independencia in the HAA 2 department), were included in this trial. After the fattening period, results obtained on 176 long cycle steers through parameters like individual final weight measured by scale, body condition (BC: scale 1-9), rib eye area (REA) and dorsal fat thickness (DFT) determined by ultrasound technique, were evaluated. The analysis of the variance included the effects type, feeding and "mouthing" (2, 4, 6 and 8 permanent teeths), with an alpha risk of 5\%. Differences on steers slaughter weight were not significant between types and feeding systems. Brangus type steers finished the fattening period with a higher $\mathrm{BC}$ and Braford type steers with a superior REA and DFT, being in both cases statistically significant in 2-4 years old steers fed on cultivated pasture.
\end{abstract}

Key words: steer, age, food, slaughter weight, ribeye area, dorsal fat thickness. 


\section{INTRODUCCIÓN}

En los últimos años hubo un marcado crecimiento ganadero en las regiones extra-pampeanas de Argentina, acompañado de una disminución del stock en la región pampeana. El nordeste argentino (NEA), segunda región ganadera del país, fue el principal beneficiado, pasando de tener el $21,5 \%$ al $27 \%$ del stock nacional. El NEA cuenta con una existencia mayor de trece millones de bovinos, en una superficie de $309.000 \mathrm{~km}^{2}$, con una relación ternero/vaca de 0,43 y una productividad desde 3-5 kg/ha/año en el oeste del Chaco a $30-50 \mathrm{~kg} /$ ha/año en Corrientes ${ }^{17,18}$.

La composición genética del ganado vacuno está compuesta en su mayoría por cebú y sus cruzas con razas europeas (Hereford y Angus), logrando razas sintéticas o compuestas adaptadas a la zona. Si bien la cría es la actividad ganadera más relevante del NEA, el incremento en la cantidad de novillitos retenidos sigue aumentando debido a por lo menos dos factores: la disminución del interés de los invernadores del sur en continuar con la actividad y la expansión de la agricultura ${ }^{1}$.

Los caracteres previos a la faena influyen en la calidad de carne y aseguran una distribución del tejido graso tanto subcutáneo como inter e intramuscular. Existen relaciones entre el tipo, sexo, edad, conformación y terminación de los bovinos y su rendimiento "al gancho", variables que son tenidas en cuenta a la hora de comprar/vender hacienda para su faena. Conocer mejor la relación que tienen estas apreciaciones visuales con el resultado posterior es de suma importancia para los agentes involucrados en el proceso productivo y comercial de hacienda para consumo $2,3,6,12,19,21$.

La alimentación determina características musculares y de "engrasamiento" que repercuten en la calidad final de la carne ${ }^{5}$. A medida que el animal se acerca al peso maduro, aumenta la proporción de grasa en el organismo. Por esta razón en ciertas condiciones de pastoreo es necesario retener 3 o 4 años los animales para poder llegar al grado de terminación deseado. Ello es debido a que la cantidad de grasa aumenta en función del peso corporal ${ }^{8}$.

Cada raza posee un peso adulto diferente, por lo cual el genotipo determina diferencias en la velocidad de desarrollo de los tejidos (razas precoces y tardías) ${ }^{11}$. Hay discrepancia con respecto a qué tipo de animal es el más eficiente. Es importante tener en cuenta las diferencias productivas y fisiológicas entre tipos y categorías, a los efectos de determinar el tipo de animal más adecuado para un propósito en particular ${ }^{8}$.

El objetivo de esta investigación fue caracterizar el tipo de novillo que llega a la faena en cuatro zonas del NEA, evaluando la influencia del tipo (Braford y Brangus), la alimentación (pastizal natural y pastura cultivada) y la edad aproximada (evaluada por número de dientes definitivos).

\section{MATERIAL Y MÉTODOS}

Los trabajos se realizaron en establecimientos de producción de carne bovina del NEA seleccionados según la "zona agroeconómica homogénea" $(\mathrm{ZAH})$ en base a las características del suelo, clima, vegetación y actividades productivas. Se incluyeron dos establecimientos de la Provincia de de Corrientes, uno ubicado en el Departamento General Paz (ZAH I) y otro en el Departamento Santo Tomé (ZAH III) ${ }^{4}$. En la Provincia de Formosa se incluyó un establecimiento del Departamento Patiño (ZAH 8: llanura central formoseña) y en la Provincia de Chaco otro ubicado en el Departamento Independencia (ZAH 2: domo central chaqueño) ${ }^{20}$.

En los establecimientos de Chaco y Formosa, el engorde se realizó bajo un sistema de pastoreo continuo sobre pastura cultivada de Panicum maximum cv Gatton Panic, con una carga animal adecuada a cada etapa de crecimiento y estación climática. En los dos establecimientos de la Provincia de Corrientes se utilizó, con igual manejo del pastoreo, un pastizal natural bajo perteneciente al área ecológica denominada lomadas arenosas y lateríticas.

Los animales utilizados fueron novillos tipos Braford y Brangus engordados en un sistema de ciclo largo y terminados para faena al superar tres años de edad como novillos pesados (más de $450 \mathrm{~kg}$ de peso vivo) en campo natural y pastura cultivada. Se evaluaron 176 novillos al final del ciclo productivo (126 y 50 tipos Braford y Brangus respectivamente), de los cuales 33 eran de Formosa (12 Braford y 21 Brangus), 81 de Corrientes (52 Braford y 29 Brangus) y 62 de Chaco (todos Braford). La recría y engorde se realizó en cada establecimiento comenzando como terneros de destete que salen del sistema de cría bovina, cuyo criterio para definir el momento de faena fue la llegada a una condición corporal superior a seis puntos que es la que busca el mercado consumidor de la región.

En cada ciclo de engorde y en cada uno de los establecimientos los animales fueron identificados y registrados al finalizar el engorde según número de caravana. Para el análisis se utilizaron los datos individuales de animales en pie, a saber: tipo (Braford y Brangus evaluados mediante caracterización fenotípica), sistema de alimentación (pastizal natural y pasturas cultivada), edad aproximada determinada por "boqueo" en el cepo (según tuvieran dos, cuatro, seis u ocho dientes incisivos definitivos se les asignaba la edad de dos, tres, cuatro y cinco años), peso vivo o peso final de faena individual medido en balanza y grado de condición corporal (CC) individual utilizando la escala americana de 1 a 9 (1 emaciada y 9 engrasada en exceso $)^{13}$.

La calidad de carcasa en pie fue evaluada por la técnica de ultrasonido, utilizando un ecógrafo Aquila Vet con transductor lineal ASP de 3,5 Mhz y $18 \mathrm{~cm}$ de peine. El transductor se ubicó en forma perpendicular al animal, utilizando el adaptador Stand Off con lubricante para mejorar la captación de las imágenes. Para el área de ojo de bife (AOB en $\left.\mathrm{cm}^{2}\right)$ la imagen se tomó 
entre las costillas $12^{\mathrm{a}}$ y $13^{\mathrm{a}}$. El espesor de grasa dorsal (EGD en $\mathrm{mm}$ ) se midió a nivel de las $3 / 4$ partes del ancho del $\mathrm{AOB}$, considerando que el inicio del AOB se encuentra próximo a la columna vertebral.

Bajo un diseño completamente aleatorizado, el análisis estadístico de las variables dependientes (peso vivo, condición corporal, área de ojo de bife y espesor de grasa dorsal) se realizó mediante análisis de la varianza para evaluar $(p<0,05)$ el efecto tipo (tipos Braford y Brangus), alimentación (pastizal natural y pastura cultivada) y "boqueo" (presencia de 2, 4, 6 y 8 dientes definitivos) en animales en pie previo a la faena. Para el análisis se utilizó el software InfoStat/Profesional ${ }^{9}$ versión 1.1 propiedad de la Facultad de Ciencias Veterinarias de la UNNE.

\section{RESULTADOS Y DISCUSIÓN}

En Tabla 1 se informan los resultados del efecto del tipo de novillo (Braford y Brangus) en los parámetros pre-faena.

El 1,6\% de peso vivo pre-faena superior en los novillos tipo Brangus no fue significativamente diferente a los tipo Braford en una experiencia anterior de nuestro grupo de trabajo (2009) realizada en novillos cruza del noroeste de la Provincia de Corrientes, en la cual tampoco fue conseguida una mayor $\mathrm{CC}$ en los novillos tipo Brangus. Las diferencias significativas en AOB a favor del tipo Braford obtenidas en este ensayo fueron similares a las logradas en un trabajo con la raza Bonsmara $\left(79,3 \mathrm{~cm}^{2}\right)$ y en cruzamientos con Angus $(50 \%$ : $71,9 \mathrm{~cm}^{2}$ y $\left.75 \%: 69,6 \mathrm{~cm}^{2}\right){ }^{15}$ y en otro ensayo con vaquillonas F1 de madres Angus con toros Criollos, donde se obtuvo menor AOB que con Hereford y Shorthon ${ }^{16}$. Otros autores obtuvieron diferencia a favor de novillos cruza Bos taurus x Bos indicus $\left(71,82 \mathrm{~cm}^{2}\right)$ respecto a Bos indicus $\left(66,38 \mathrm{~cm}^{2}\right)$ con 450 y $470 \mathrm{~kg}$ de peso de faena respectivamente ${ }^{14}$. En discordancia, otros tipos como novillos Brangus 7/8, Brangus 3/8, Braford 3/4 y Braford $1 / 2$ no registraron diferencias de $\mathrm{AOB}$ debidas al tipo en el NEA ${ }^{10}$.

En cambio, en el presente ensayo, los valores de EGD superiores en novillos tipo Brangus, fueron muy inferiores a los logrados por otros autores que obtuvieron en novillos Hereford 17,1 mm y en Angus 18,3 mm respecto a los restantes, Bonsmara puro y con 50 y $25 \%$ de Angus (16,0; 14,9 y 16,3 mm respectivamente) ${ }^{15}$.

Los resultados del efecto del sistema de alimentación de novillos sobre los parámetros pre-faena se informan en Tabla 2. La alimentación no influyó en el peso de faena de novillos tipos Braford y Brangus, en coincidencia con lo informado por otros autores en novillos producidos en pasturas y pasturas más suplementación mineral y mineral con proteínas ${ }^{14}$, aunque sí en el grado de CC, lográndose en pasturas cultivadas $0,31 \mathrm{~mm}$ más de espesor de grasa dorsal, difiriendo a lo comprobado midiendo la tasa de engrasamiento en sistemas más controlados de terneros recriados a corral y en pastura de Agropiro ${ }^{7}$. El AOB fue superior
Tabla 1. Resultados pre-faena obtenidos para cada variable de ambos tipos.

\begin{tabular}{llccc}
\hline tipo & variable & $\mathrm{n}$ & media & $\mathrm{DE}$ \\
\hline \multirow{4}{*}{ Braford } & PV kg & 126 & $503,67 \mathrm{a}$ & 41,52 \\
& CC 1-9 & 125 & $5,84 \mathrm{~b}$ & 1,28 \\
& AOB cm & 126 & $64,36 \mathrm{a}$ & 9,54 \\
& EGD mm & 126 & $0,95 \mathrm{a}$ & 0,35 \\
\hline \multirow{5}{*}{ Brangus } & PV kg & 50 & $511,78 \mathrm{a}$ & 59,47 \\
& CC 1-9 & 50 & $7,47 \mathrm{a}$ & 0,56 \\
& AOB cm & 50 & $60,44 \mathrm{~b}$ & 10,19 \\
& EGD mm & 50 & $0,73 \mathrm{~b}$ & 0,14 \\
\hline
\end{tabular}

n: número de observaciones, DE: desvío estándar, PV: peso vivo, CC: condición corporal, $\mathrm{AOB}$ : área de ojo de bife, EGD: espesor de grasa dorsal. Letras distintas indican diferencias significativas entre los promedios estimados (test de Tukey, $\mathrm{p}<0,05)$.

Tabla 2. Influencia de la alimentación en las variables pre-faena de ambos tipos.

\begin{tabular}{llccc}
\hline alimentación & variable & $\mathrm{n}$ & media & $\mathrm{DE}$ \\
\hline \multirow{4}{*}{ campo } & PV kg & 81 & $505,22 \mathrm{a}$ & 49,43 \\
natural & CC 1-9 & 80 & $5,74 \mathrm{~b}$ & 1,47 \\
& AOB cm & 81 & $57,23 \mathrm{~b}$ & 7,57 \\
& EGD mm & 81 & $7,2 \mathrm{~b}$ & 0,18 \\
\hline \multirow{4}{*}{ pasturas } & PV kg & 95 & $506,61 \mathrm{a}$ & 45,62 \\
cultivadas & CC 1-9 & 95 & $6,78 \mathrm{a}$ & 1 \\
& AOB cm & 95 & $68,37 \mathrm{a}$ & 8,63 \\
& EGD mm & 95 & $10,3 \mathrm{a}$ & 0,35 \\
\hline
\end{tabular}

n: número de observaciones, DE: desvío estándar, PV: peso vivo, CC: condición corporal, AOB: área de ojo de bife, EGD: espesor de grasa dorsal. Letras distintas indican diferencias significativas entre los promedios estimados (test de Tukey, $\mathrm{p}<0,05)$.

en pasturas cultivadas, contrariamente a los resultados obtenidos en novillitos Angus en pastoreo vs confinamiento durante la recría y terminación en la región central del país ${ }^{22}$.

En la Tabla 3 se presentan los resultados del efecto de la edad ("boqueo") de novillos tipo Braford y Brangus sobre los parámetros pre-faena. El 76\% de los novillos se terminaron como novillos pesados con 4 y 6 dientes y un peso vivo que fue superior para los animales de 2-4 dientes versus los de 6-8 dientes, con diferencias en $9 \mathrm{~kg}$ menos (entre los novillos de 4 y 2 dientes), $27 \mathrm{~kg}$ más (entre 6 y 4 dientes) y $19 \mathrm{~kg}$ más (entre 8 y 6 dientes), lo que corresponde a una edad aproximada de 3 a 4 años, y un peso vivo mayor a medida que se incrementa la edad. La CC solo fue diferente al alcanzar el estadio de ocho dientes. El número de dientes definitivos no influyó en el AOB ni el EGD de novillos tipo Braford y Brangus producidos en el NEA.

En conclusión, surge que el peso de faena de los novillos tipos Braford y Brangus no se diferenció estadísticamente, en cambio los del tipo Brangus finalizaron el engorde con una condición corporal superior y 
Tabla 3. Efectos de la edad en los parámetros prefaena de ambos tipos.

\begin{tabular}{llccc}
\hline "boqueo" & variable & $\mathrm{n}$ & media & $\mathrm{DE}$ \\
\hline \multirow{4}{*}{ dos } & PV kg & 6 & $494,67 \mathrm{a}$ & 34,29 \\
dientes & CC 1-9 & 6 & $5,5 \mathrm{a}$ & 0,77 \\
& AOB cm ${ }^{2}$ & 6 & $66,18 \mathrm{a}$ & 5,38 \\
& EGD mm & 6 & $8,1 \mathrm{a}$ & 0,28 \\
\hline \multirow{4}{*}{ cuatro } & PV kg & 64 & $485,83 \mathrm{a}$ & 33,93 \\
dientes & CC 1-9 & 63 & $5,95 \mathrm{a}$ & 1,38 \\
& AOB cm ${ }^{2}$ & 64 & $63,22 \mathrm{a}$ & 8,25 \\
& EGD mm & 64 & $8,5 \mathrm{a}$ & 0,29 \\
\hline \multirow{4}{*}{ seis } & PV kg & 70 & $512,46 \mathrm{ab}$ & 47,43 \\
dientes & CC 1-9 & 70 & $6,26 \mathrm{a}$ & 1,34 \\
& AOB cm & 70 & $64,07 \mathrm{a}$ & 11 \\
& EGD mm & 70 & $9,8 \mathrm{a}$ & 0,36 \\
\hline \multirow{4}{*}{ ocho } & PV kg & 36 & $531,06 \mathrm{~b}$ & 54,83 \\
dientes & CC 1-9 & 36 & $7,15 \mathrm{~b}$ & 0,89 \\
& AOB cm & 36 & $61,19 \mathrm{a}$ & 10,67 \\
& EGD mm & 36 & $7,9 \mathrm{a}$ & 0,26 \\
\hline
\end{tabular}

n: número de observaciones, DE: desvío estándar, PV: peso vivo, CC: condición corporal, AOB: área de ojo de bife, EGD: espesor de grasa dorsal. Letras distintas indican diferencias significativas entre los promedios estimados (test de Tukey, $\mathrm{p}<0,05)$.

los tipo Braford con mayores valores para el área de ojo de bife y espesor de grasa dorsal. Las variables correspondientes a campo natural versus pasturas cultivadas tampoco influyeron estadísticamente en el peso final o de faena, pero sí en la condición corporal, área de ojo de bife y espesor de grasa dorsal, las que fueron superiores sobre pasturas cultivadas. El avance de la edad de los novillos influye favorablemente en el peso vivo y la condición corporal, los cuales aumentan, no así en el área de ojo de bife y espesor de grasa dorsal.

Agradecimientos. A los alumnos de la Facultad de Ciencias Veterinarias de Corrientes (Argentina), personal de campo, propietarios de los establecimientos, jóvenes colegas, becarios y asesores técnicos que nos acompañaron en las tareas de conducción y recolección de datos, imprescindibles para poder obtener los resultados presentados.

\section{REFERENCIAS}

1. Arias Mañotti AA. 2007. Panorama general de la ganadería en los diferentes ambientes del NEA, Ed. Amanecer Rural, Resistencia (Chaco, Argentina), p. 6.

2. Boccard R, Nauder RT, Cronje DE, Smith MC, Venter HJ, Rossow EJ. 1979. The influence of age, sex and breed of cattle on their muscle characteristics. J. Meat Sci 3: 261280.

3. Bognio G, Begenisic M, Moronta P. 2006. Rendimiento en carne de las reses en relación al biotipo de los animales. http:// www.produccion-animal.com.ar.
4. Calvi M, Acosta F. 2005. Provincia de Corrientes: zonas agroeconómicas homogéneas. Serie Técnica EEA INTA Mercedes $\mathrm{N}^{\circ} 36$ (Corrientes, Argentina), $75 \mathrm{p}$.

5. Cañeque V, Sañudo C. 2000. Metodología para el estudio de la calidad de la canal y de la carne en rumiantes. Publ. INIA (Ministerio de Ciencia y Tecnología), Madrid, $67 \mathrm{p}$.

6. Cañeque V, Sañudo C. 2005. Estandarización de las metodologías para evaluar la calidad del producto (animal vivo, canal carne y grasa) en los rumiantes. Publ. INIA (Ministerio de Ciencia y Tecnología), Madrid, Serie $\mathrm{N}^{\mathrm{o}} 3$, p. 313-321.

7. Ceconi I, Russi JP, Davies P, Méndez D. 2009. Efecto de alimentación sobre la performance productiva de novillos británicos engordados a corral. Rev Arg Prod Anim 29: 232-233.

8. Di Marco O. 2006. Crecimiento de vacunos para carne, Ed. INTA, Balcarce (Argentina), 204 p.

9. Di Rienzo JA, Casanoves F, Balzarini MG, González L, Tablada M, Robledo CW. 2008. InfoStat, versión 2008, Grupo InfoStat, FCA, Universidad Nacional de Córdoba, Argentina. $336 \mathrm{p}$.

10. Fernández Madero J, Berti RN, Moisá S, Sühring S, Orellana CR. 2009. Determinación del área de ojo de bife de la carne de novillos y toros de diferentes biotipos bovinos del NOA. Rev Arg Prod Anim 29: 148-150.

11. Kempster AJ, Croston D, Jones DW. 1981. Value of conformation as an indicator of sheep carcass composition within and between breeds. J Anim Prod (Ankara, Turquía) 23: 39-49.

12. Lawrie RA. 1998. Ciencia de la carne, $3^{\mathrm{a}}$ ed., Acribia, Zaragoza, $367 \mathrm{p}$.

13. Lowman BG, Scott NA, Somerville SH. 1976. Condition scoring of cattle. Bull Edinburgh School Agric (Scotland) 6: 31 .

14. Moreira FB, Evelázio NE, Matsushita M, Nunes I, Gonçalves W. 2003. Evaluation of carcass characteristics and meat chemical composition of Bos indicus and Bos indicus $x$ Bos taurus crossbred steers finished in pasture systems. Braz Arch Biol Technol 26: 609-616.

15. Pordomingo AJ, Grigioni G, Carduza F, Pordomingo AB, Pini F, Masgoret S. 2009. Efectos del cruzamiento con Bonsmara sobre novillos en confinamiento. Aumento de peso y parámetros de confinamiento. Rev Arg Prod Anim 29: 116-117.

16. Pordomingo AJ, Grigioni G, Carduza F, Irurueta $\mathbf{M}$, Lernoud P, Volpi G, Pordomingo AB. 2009. Carne de vaquillonas F1, Criollo, Hereford o Shorthorn x Angus terminadas en pastoreo. Perfil físico. Rev Arg Prod Anim 29: 125-126.

17. Rearte, D. 2007. La producción de carne en Argentina. Publ. INTA. http://inta.gov.ar/Balcarce/carnes/prodcarne. htm.

18. Rearte D. 2010. Situación actual y prospectiva de la producción de carne vacuna. http://www.inta.gov.ar/balcarce/ carnes/SituacionActual_Prostpectiva_Produccion_carnevacuna.pdf. 
19. Renerre M, Dumont F, Gatellier P. 1996. Antioxidant enzyme activities in beef in relation to oxidation of lipid and myoglobin. J Meat Sci 43: 109-111.

20. Torriglia I. 2007. Identificación y caracterización de los sistemas de producción de la región Chaco-Formosa. Docum. Centro Reg. Chaco-Formosa INTA, 15 p.

21. Touraille C, Girard JP. 1985. Influence du sexe et de l'âge à l'abattage sur les qualities organoleptiques des viandes de bovins Limousins abattus entre 16 et 33 mois. Bull Tech CRZV-Inst Nat Rech Agron (Theix, France) 60: 83-97.

22. Volpi G, Pordomingo AJ, Griguioni G, Carduza F, Irurueta M. 2009. Parámetros físicos y sensoriales de carne bovina con diferentes sistemas de recría y terminación. Rev Arg Prod Anim 29: 115-116.

\section{Asociación Cooperadora de la Facultad de Ciencias Veterinarias Universidad Nacional del Nordeste}

Personería Jurídica No 647/92 y 912/00

Sargento Cabral 2139

3400 Corrientes

Argentina

La Asociación Cooperadora de la Facultad de Ciencias Veterinarias de la UNNE fue constituida el 10 de diciembre del año 1991 como entidad de bien público, con el objeto de promover y coadyuvar las actividades científicas, educativas y culturales relacionadas con las ciencias veterinarias. En tal sentido, implementa acciones para colaborar con la enseñanza, extensión, actualización y difusión científica que realiza dicha casa de estudios.

\section{Beneficios que brinda a sus asociados:}

- Fotocopias con descuentos especiales del $20 \%$ en la Fotocopiadora Copias.com que funciona dentro del predio de la Facultad de Ciencias Veterinarias.

- Descuentos del 10\% para la adquisición de libros de la Editorial Inter-Médica.

- Descuentos especiales en otros rubros.

Sea solidario, asóciese en beneficio de toda la comunidad universitaria

Tel. 03783-425753 interno 186• Página Web www.vet.unne.edu.ar•E-mail: cooperadora@vet.unne.edu.ar 